\title{
Modelling of chromatin morphologies in breast cancer cells undergoing apoptosis using generalized Cauchy field.
}

\begin{abstract}
Chromatin morphologies in human breast cancer cells treated with an anti-cancer agent are analyzed at their early stage of programmed cell death or apoptosis. The gray-level images of nuclear chromatin are modelled as random fields. We used two-dimensional isotropic generalized Cauchy field to characterize local self-similarity and global long-range dependence behaviors in the image spatial data. Generalized Cauchy field allows the description of fractal behavior inferred from fractal dimension and the long-range dependence inferred from correlation exponent to be carried out independently. We demonstrated the usefulness of locally self-similar random fields with long-range dependence for modelling chromatin condensation.
\end{abstract}

Keyword: Apoptosis; Fractal dimension; Locally self-similar processes; Long- 